\title{
Effect of Vitex agnus castus L. essential oil on stereological architecture of adrenal glands in female mice
}

\author{
Gholamreza Hamidian $^{a^{*}}$, Razagh Mahmoodi ${ }^{b}$
}

${ }^{a}$ Department of Basic Science, Faculty of Veterinary Medicine, University of Tabriz, Tabriz, Iran

${ }^{b}$ Department of Food Hygiene, Faculty of Veterinary Medicine, University of Tabriz, Tabriz, Iran

\section{ARTICLE INFO}

\section{* Correspondence to:}

Gholamreza Hamidian

Department of Basic Science, Faculty of Veterinary Medicine,

University of Tabriz, Tabriz, Iran

e-mail: hamidian@tabrizu.ac.ir

\section{Keywords:}

Adrenal

Essential oil

Phytosterogen

Stereology

Vitex agnus

\section{ABSTRACT}

The adrenal gland is of critical importance for a plethora of biological processes and has a pivotal role in different stages of life. The structure and function of adrenal can be affected by steroid hormones. Vitex agnus-castus L. (VAC) is a phytosterogen shrub and has been used in the treatment of many female conditions such as menstrual disorders in traditional medicine. So, this study was conducted to investigate the effects of VAC essential oil on structure of adrenal glands. In this study 20 young adult female BALB/c mice were randomly divided into four groups: group 1 as control group, group 2 received $75 \mathrm{mg} / \mathrm{kg}$ VAC, group 3 received $150 \mathrm{mg} / \mathrm{kg}$ VAC and group 4 which received $300 \mathrm{mg} /$ $\mathrm{kg}$ VAC essential oil via gastric gavage for seven consecutive days. At the eighth day, animals were euthanized and the adrenal glands were quickly removed, weighted and fixed in buffered formalin. The samples were processed by routine and standard paraffin embedding and serially sectioned and stained by H\&E. The total volume of adrenal gland, adrenal cortex and cortical zones were estimated by Cavalieri's point-counting principle using Weibel's multipurpose test grid $\mathrm{M}_{42}$. Total number of cortical cell in each zona was estimated by unbiased stereological methods. The results showed that adrenal gland mass were significantly decreased in group 4 . It was also found that there are no significant differences in the zona glomerulosa volume and cell number among examined groups. The volume of cortex, zona fasciculata, zona reticularis and cell number of these regions were significantly reduced in group 4 compared to other groups $(\mathrm{P}<0.05)$. Finally, it can be concluded that VAC essential oil can affected adrenocortical structure. So, it should be considered with caution with high doses in traditional medicine.

J. Exp.Clin.Med., 2014; 31:126 